Article

\title{
Junior Secondary School Students' Conceptions of and Approaches to Learning Mathematics and Their Relationships in Mainland China
}

\author{
Xinrong Yang ${ }^{1, *(\mathbb{D}}$, Frederick Leung ${ }^{1,2}$ and Shasha Zhang ${ }^{1}$ \\ 1 School of Mathematics and Statistics, Southwest University, Chongqing 400715, China; \\ frederickleung@hku.hk (F.L.); shasha_zhang603@126.com (S.Z.) \\ 2 Faculty of Education, The University of Hong Kong, Hong Kong, China \\ * Correspondence: xinrong.yang@yahoo.com; Tel.: +86-23-6825-2350
}

Received: 18 February 2019; Accepted: 26 April 2019; Published: 27 April 2019

\begin{abstract}
This study reports the findings of a study which investigated junior secondary school students' conceptions of and approaches to learning mathematics and their relationships in Mainland China. Two questionnaires, conceptions of learning mathematics (COLM) and approaches to learning mathematics (ALM), were administered to 1590 students. Descriptive analysis results suggest that Chinese junior school students tend to hold (1) higher-level conceptions of learning mathematics rather than lower-level conceptions, and (2) deep approaches to learning mathematics with a rather mixed surface and deep motive in learning mathematics. Correlation and regression analysis results confirm a structural relationship between students' conceptions of and approaches to learning in the subject of mathematics. Two factors of students' lower-level conceptions of learning mathematics, "memorizing" and "testing", were the strongest predictor for the surface approaches to mathematics learning, while students' higher-level conceptions of learning mathematics, such as "applying" and "understanding and mathematical thinking", had a noticeable effect on their deep approaches to learning mathematics. However, under the pressure of examination in Mainland China, "understanding and mathematical thinking" was also found to exert quite a strong influence on students" "surface motive".
\end{abstract}

Keywords: conceptions of learning; approaches to learning; mathematics learning; junior secondary school

\section{Introduction}

Since the 1990s, numinous international comparative studies (e.g., PISA and TIMSS) have consistently found that Chinese students, including students from Mainland China, outperform their Western counterparts in achievements in mathematics [1,2]. However, researchers also found that the Chinese learning environment, characterized by factors such as large class sizes, expository teaching methods, and examination-driven curricula [3,4], is "impoverished" [5] according to Western criteria. Such a paradoxical situation has been identified as the "Chinese Learner Paradox" in the literature [5] and there has been a great deal of academic research interest in seeking a possible explanation to the paradox. Factors such as culture [3], the number system and language [6], parental involvement [7], and classroom instruction [4] have been proposed in previous studies to explain the paradox.

Such factors (e.g., teaching approach and learning resources), however, will only indirectly relate to students' achievements in mathematics [5,8]. Factors from students themselves, such as their conceptions of learning mathematics and the approaches they employed to learn mathematics, will more directly and closely relate to students' learning outcomes $[9,10]$. Therefore, to achieve a fuller and deeper understanding of the "Chinese Learner Paradox" in general and Chinese students' achievements 
in mathematics in particular, information from students should be investigated systematically and specifically. Indeed, mathematics plays a fundamental role in students' sustainable development. Therefore, a solid foundation in school mathematics, even starting from the kindergarten stage, will facilitate the development of mathematical skills and interdisciplinary and transdisciplinary skills in later grades and in students' future life as well [11,12]. Unfortunately, to date, very few studies have tried to investigate the reasons from the side of students for the interpretation of Chinese students' success in mathematics, such as their conception of learning and approaches to learn mathematics in the field of mathematics education in Mainland China [10].

The students' conception of learning, however, has been identified as academic or school domain-specific and culturally and contextually dependent $[13,14]$. Therefore, the impact of a specific schooling subject and culture on learning and conceptions of learning implies that students from Mainland China may hold different conceptions of learning mathematics and employ different approaches to learning mathematics. Moreover, culture is not static but is ever-changing [15] (p. 3-31). For the mathematics education culture in Mainland China, there has been quite a significant change since the beginning of this century, as a new wave of mathematics curriculum reform started in 2001. A fundamental and influential change of the new mathematics curriculum is shifting away from transmitting knowledge from teachers to the construction of knowledge by students, with the enhancement of connections between mathematics and real-life applications [4].

Such reformed mathematics curriculum ideas should have gradually formed a new mathematics education culture in Mainland China, which will in turn gradually change students' conceptions of and approaches to mathematics learning. However, "there is no systematic work to date that examines the influence of the changing educational context on the Chinese learner" [15] (p. 12). Until now, Chinese students' conceptions of and approaches to mathematics learning under the changing educational context remain unknown. In view of this, the present study aims to investigate junior secondary school students' conceptions of and approaches to mathematics learning in Mainland China and the relationship between these two constructs.

\section{Literature Review}

\subsection{Conceptions of (Mathematics) Learning}

Conceptions of learning refer to students' views about their learning experiences and their preferred ways of undertaking the learning process [16,17]. The pioneering work investigating students' conceptions of learning was conducted by Saljo [18], who identified five different categories: (1) increasing of knowledge; (2) memorizing; (3) acquisitions of facts, procedures that can be retained and/or utilized in practice; (4) abstraction of meaning; and (5) an interpretative process aimed at the understanding of reality. Following this study, many researchers have investigated conceptions of learning held by students in different educational contexts and subject domains $[14,19,20]$.

Findings identified in these later studies suggest that students' conceptions of learning are socially and culturally dependent $[13,14]$. For example, Tsai identified seven categories of learning science held by high school students in Taiwan: (1) memorizing, (2) testing, (3) calculating and practicing problems, (4) increasing knowledge, (5) applying, (6) understanding, and (7) seeing problems in a new way [14]. Among these, the conception of "testing" is closely related to the widely mentioned traditional Chinese examination culture in Taiwan [14].

Furthermore, previous researchers have suggested that students' conceptions of learning follow a hierarchical order [21]. For example, Tsai divided the above-mentioned seven categories of conceptions into two groups: lower-level and higher-level conceptions [14]. The lower-level conceptions, which include "memorizing", "testing" and "calculating and practicing", represent a passive and fragmented view of learning which regards learning as a process of copying and reproducing information which needs to be learned $[13,22]$. The high-level conceptions of learning include increasing knowledge, applying, understanding and seeing problems in a new way. This is similar to a 
constructive view of learning, and it emphasizes understanding and meaning-making by connecting new information to prior knowledge and students' application of knowledge [23].

More recently, researchers have argued for the domain-specific attribute of conceptions of learning $[10,14]$. In the field of mathematics education, a few studies have tried to investigate students' conceptions of learning mathematics specifically $[10,20]$. Their findings suggest the existence of similarities between students' conceptions of learning mathematics and students' conceptions of learning in general or in science-related subjects, such as the emphasis on understanding and the acquirement of knowledge. However, differences were also identified in these studies. One major difference is the emphasis of the acquirement of a mathematical way of thinking and understanding of mathematics, which may be unique to the subject of mathematics.

\subsection{Students' Approaches to (Mathematics) Learning}

Students' approaches to learning refer to the ways in which students go about their learning or processing of their academic tasks [9]. Over the past decades, previous researchers have generally found that students tend to approach their learning in two ways: a deep approach and surface approach [24]. The surface approach to learning is based on students' external interest or extrinsic motivation to conduct rote learning, such as focusing on the concrete and literal aspects of the task's components rather than on their meaning [24,25]. In contrast, the deep approach is based on students' inner-interest or intrinsic motivation, with an intention to seek to maximize their understanding of the underlying meaning of the learning material [22]. Students who utilize the deep approach will also intend to seek to integrate components of the task and with other tasks as well [25].

Researchers also argued that approaches to learning are "not immune to the learning context" [26]. Indeed, differences between students' learning approaches in different contexts have been identified in previous studies [27]. In addition, previous researchers have pointed out that the essence of surface and deep approaches to learning varies across different disciplines [28]. For example, Chiou et al. described that surface approaches to learning mathematics may refer to the processes of repeatedly calculating or following a certain problem-solving procedure, while surface approaches to learning biology may refer to the processes of matching the name of a specific species with its features [10].

\subsection{Relationship between Students' Conception of and Approaches to (Mathematics) Learning}

During the past years, researchers have investigated the relationship between students' conceptions of and approaches to learning in various subject domains, such as science $[22,29]$ and computer technology [30], and in various educational contexts, such as in Portugal [26] and Taiwan [29]. The trend of the findings in previous studies is that students with lower-level conceptions of learning are more likely to use surface approaches to learning, while students possessing higher-level conceptions of learning tend to adopt deep approaches to learning. For example, Chiou et. al. found students' higher-level learning conceptions such as "seeing problems in a new way" are more likely to positively correlate with their deep approaches to learning physics, whereas their lower-level conceptions of learning such as "testing" are more likely to correlate with their surface approaches and negatively predict students' deep approaches [22].

However, several inconsistent findings were also identified in previous studies. For example, Li et. al. found that the component of students' lower-level conceptions of learning, "learning chemistry by memorizing", could positively predict a deep motive in learning, while the component of higher-level conceptions of learning, "learning chemistry by transforming", was positively correlated to surface motives in learning chemistry [28]. Researchers argued that this kind of inconsistency may be caused by the differences in contexts and subject areas [29]. In other words, since students perform differently in different learning domains and educational contexts [24], the relationship between students' conceptions of learning and approaches to learning may be also different in different subject domains.

However, to date, there have been hardly any empirical studies which have focused on students' conceptions of and approaches to learning mathematics and their relationships in Mainland China, 
in which the educational environment is now gradually changing to be more constructivist-oriented [4]. To fill this research gap, this study was undertaken to investigate the following questions:

(1) the characteristics of junior secondary school students' conceptions of and approaches to mathematics learning in Mainland China;

(2) the relationship between Chinese junior secondary school students' conceptions of mathematics learning and their approaches to learning mathematics.

\section{Method}

\subsection{Participants}

Taking into account factors such as school academic background, school location (rural and urban), class size and mathematics teachers' teaching experience, 1590 Grade 7 and Grade 8 (at the age of 13 or 14) students at the junior secondary school level in Mainland China were chosen for the present study. The students were from 45 classrooms and 18 coeducational schools instead of single-sex schools in 7 provinces located in various parts of Mainland China, namely Sichuan, Chongqing, Shan'xi, Guangdong, Hunan, Tianjin, and Liaoning, which represent a wide range of economic and educational development levels. Table 1 provides detailed background information about the participants of the study.

Table 1. Background characteristics of the participants.

\begin{tabular}{ccc}
\hline Subgroup & Frequency (N) & Percentage (\%) \\
\hline Gender & & \\
Boys & 770 & 49.4 \\
Girls & 789 & 50.6 \\
Grade & & \\
7 & 700 & 44.0 \\
8 & 890 & 56.0 \\
School location & & \\
Urban & 870 & 54.7 \\
Rural & 720 & 45.3 \\
\hline
\end{tabular}

Note: 31 students did not indicate their gender in the survey.

\subsection{Instruments}

To investigate junior secondary school students' conceptions of and approaches to learning mathematics, two questionnaires were employed in this study. A five-point Likert-type response scale ( $5=$ strongly agree to $1=$ strongly disagree) was used in the questionnaires. A brief description of the two questionnaires is seen below.

The conceptions of learning mathematics questionnaire (COLM): This questionnaire was modified from Lee et al.'s [16] questionnaire, which was originally designed in Taiwan to assess students' conceptions of learning science. This questionnaire has been widely adapted and validated in various school subjects and schooling levels, including mathematics [31]. The original questionnaire contains seven scales, namely "memorizing", "testing", "calculating and practicing", "increasing knowledge", "applying", "understanding", and "seeing problems in a new way". The first three scales are regarded as lower-level conceptions, while the latter four scales are considered to be higher-level of conceptions of learning [16].

Based on the Chinese version of the questionnaire, items in the first six scales were modified according to the mathematics education tradition in Mainland China. In addition, since training students to think mathematically and developing students' mathematics thinking is a main goal for the mathematics curriculum, the last dimension, "seeing problems in a new way", was modified as "seeing things mathematically and mathematical thinking". Items such as "learning mathematics 
means finding a better way to explain things and phenomena mathematically" were modified from the original items. New items such as "learning mathematics means developing mathematical thinking which can be used to help me view the world mathematically" were added to meet the aims of this dimension.

To enhance the suitability of the modified COLM for Mainland China, a university mathematics education researcher and two highly-experienced junior school mathematics teachers were invited to examine the content of all the items. Relevant modifications were further made according to their comments. Each scale included four to seven items in the final version of the questionnaire, and the final COLM contains 42 items in total. Table 2 provides a detailed description of what each scale measures and an example item.

Table 2. Description and sample item for each scale in the modified conceptions of learning mathematics (COLM) questionnaire.

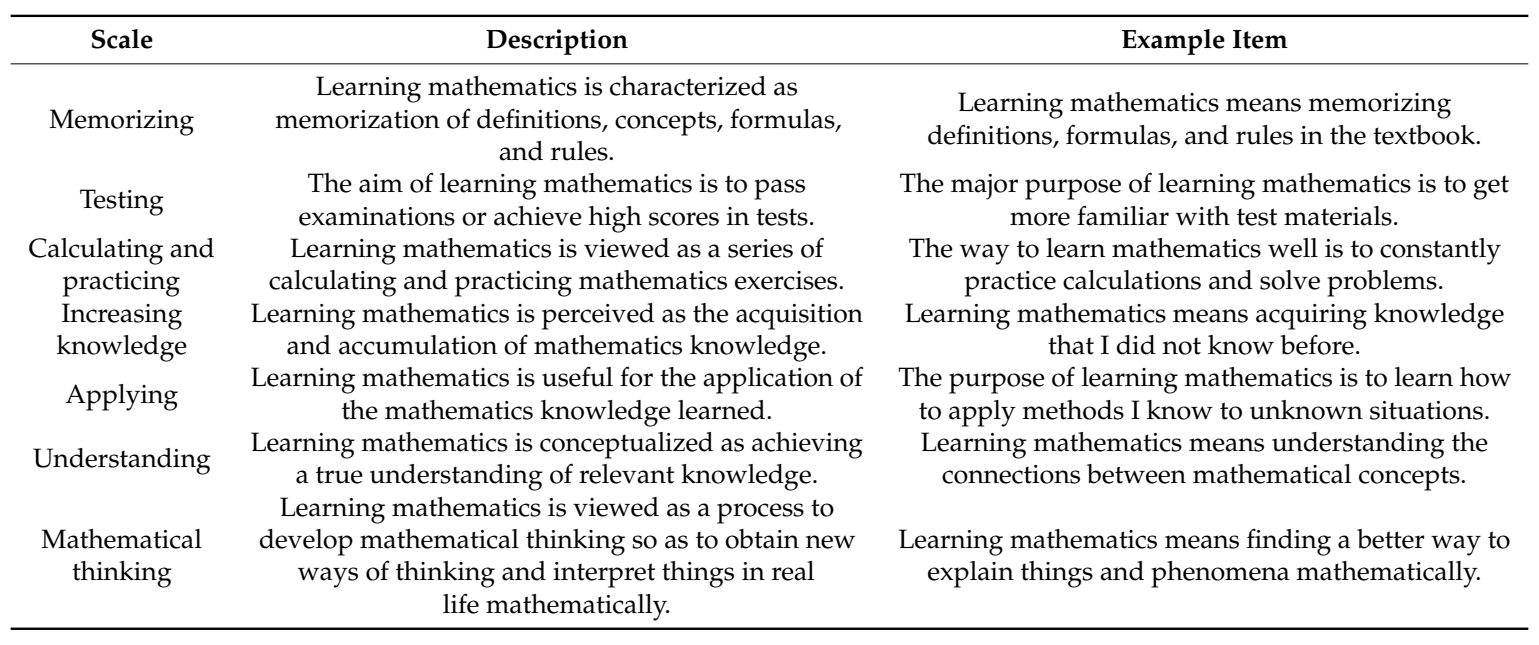

Students' approaches to learning mathematics questionnaire (ALM): The ALM was modified from the questionnaire designed by [16], which was modified from the revised learning process questionnaire (R-LPQ-2F) by Kember et al. [24]. The R-LPQ-2F consists of two factors, deep approaches and surface approaches, and each factor includes two sub-scales, namely motive and strategy. Thus, the modified ALM in the study also consists of four scales. The modified ALM was reviewed by the mathematics education researcher and two mathematics teachers to confirm its content validity. A brief description of each of the four scales (6-9 items for each scale and 29 items in total), each with a sample item, is presented below:

(1) Deep motive: learning mathematics is fostered by students' inner interests and intrinsic motivation. Example item: I work hard at studying mathematics because I find it is very interesting;

(2) Deep strategy: mathematics is learned with the use of more meaningful strategies. Example item: I try my best to understand the meaning of the content I read in the textbook;

(3) Surface motive: mathematics learning is driven by extrinsic motivation. Example item: I want to do well in mathematics so that I can please my parents;

(4) Surface strategy: mathematics is learned by using rote-like strategies. Example item: I find that the best way to pass mathematics examinations is to try to remember the answers or procedures to likely questions.

\subsection{Data Analysis}

Firstly, exploratory factor analysis (EFA) and confirmatory factor analysis (CFA) were performed to examine the construct validity of the two modified questionnaires. The item mean score of each scale with the standard deviation of each scale were then computed to obtain a sample description 
of students' conceptions of and approaches to mathematics learning. Pearson correlation analysis was conducted to examine the interrelationships between students' conceptions of and approaches to mathematics learning. Finally, a stepwise regression model was built with the use of all the factors of COLM as predictors, and each factor of ALM was viewed as an outcome variable.

\section{Findings}

\subsection{Validation of the Modified COLM and ALM Questionnaires}

Exploratory factor analysis (EFA) and confirmatory factor analysis (CFA) were performed to examine the factor structure and the reliability of the factors in these two modified questionnaires. The participants $(n=1590)$ were randomly split into two subsets for the EFA $(n=762)$ and the CFA $(\mathrm{n}=828)$, respectively.

For COLM, principal components factor analysis (with varimax rotation) was utilized to confirm the structure, and six factors (rather than seven factors) were identified. The original scales of "understanding" and "seeing things mathematically and mathematical thinking" came together to form one scale, labeled as "understanding and mathematical thinking". As a result of the analyses, 25 items from the modified COLM were retained. The result of the final factor analysis is presented in Table 3, which shows that the factor loading of each item is greater than 0.4 for the relevant factor and less than 0.4 for all other factors. These factors accounted for $56.71 \%$ of the variance.

Table 3. Factor loadings, Cronbach $\alpha$ values, factor means and standard deviations for COLM.

\begin{tabular}{|c|c|c|c|c|c|c|}
\hline \multirow[b]{2}{*}{ Item No. } & \multicolumn{6}{|c|}{ Factor Loading } \\
\hline & Memorizing & Testing & $\begin{array}{l}\text { Calculating and } \\
\text { Practicing }\end{array}$ & Knowledge & Applying & $\begin{array}{l}\text { Understanding and } \\
\text { Mathematical } \\
\text { Thinking }\end{array}$ \\
\hline 1 & 0.736 & & & & & \\
\hline 2 & 0.673 & & & & & \\
\hline 3 & 0.670 & & & & & \\
\hline 4 & 0.656 & & & & & \\
\hline 5 & 0.600 & & & & & \\
\hline 6 & & 0.771 & & & & \\
\hline 7 & & 0.678 & & & & \\
\hline 8 & & 0.657 & & & & \\
\hline 9 & & 0.630 & & & & \\
\hline 10 & & 0.465 & & & & \\
\hline 11 & & & 0.767 & & & \\
\hline 12 & & & 0.650 & & & \\
\hline 13 & & & 0.623 & & & \\
\hline 14 & & & & 0.694 & & \\
\hline 15 & & & & 0.603 & & \\
\hline 16 & & & & 0.584 & & \\
\hline 17 & & & & & 0.774 & \\
\hline 18 & & & & & 0.520 & \\
\hline 19 & & & & & & 0.740 \\
\hline 20 & & & & & & 0.723 \\
\hline 21 & & & & & & 0.690 \\
\hline 22 & & & & & & 0.676 \\
\hline 23 & & & & & & 0.662 \\
\hline 24 & & & & & & 0.608 \\
\hline 25 & & & & & & 0.585 \\
\hline$\alpha$ & 0.78 & 0.74 & 0.65 & 0.64 & 0.50 & 0.83 \\
\hline Item Mean \pm SD & $2.86 \pm 0.86$ & $2.81 \pm 0.89$ & $3.60 \pm 0.86$ & $3.47 \pm 0.85$ & $3.56 \pm 0.87$ & $3.61 \pm 0.74$ \\
\hline
\end{tabular}

The remaining 25 items were further validated by confirmatory factor analysis (CFA). No items were deleted during the CFA, the results of which indicated a good fit with GFI, IFI, TLI, and CFI, all of which were above $0.90(\mathrm{GFI}=0.935$, IFI $=0.918, \mathrm{TLI}=0.902, \mathrm{CFI}=0.918)$, and the RMSEA was below 0.05 (RMSEA $=0.048$ ). The alpha coefficient for each of the six identified factors was around $0.50-0.83$, 
and the overall alpha coefficient was 0.86 , suggesting a sound reliability of the modified COLM in Mainland China.

For students' conceptions of learning mathematics, as shown in Table 3, relatively speaking, students attained high mean scores for "increasing knowledge", "applying", and "understanding and mathematical thinking". Their average scores for "memorizing" and "testing" were lower in comparison to those of the other factors. However, the mean score for "calculating and practicing", an originally lower-level conception of learning, is the highest among the six factors. These results seem to suggest that current Chinese junior secondary school students tend to hold higher-level conceptions of learning mathematics, but with a strong conception of "calculating and practicing".

Similarly, exploratory factor analysis was also used to validate the factors of ALM. Table 4 shows the final results. As shown in Table 4, all the four original factors were identified, and for each factor, the factor loadings of all the retained items were greater than 0.4 . In total, 17 items were retained and these factors explained $54.58 \%$ of the variance. The remaining 17 items were further validated by CFA. No items were deleted during the CFA, the results of which indicated a good fit with GFI, IFI, TLI, and CFI, all of which were above $0.90(\mathrm{GFI}=0.947$, IFI $=0.925$, TLI $=0.901, \mathrm{CFI}=0.925)$, and the RMSEA was below 0.06 (RMSEA $=0.057$ ). The alpha coefficient for each factor ranged from 0.67 to 0.81 , and the overall alpha coefficient was 0.80 , indicating satisfactory internal consistency.

Table 4. Factor loadings, Cronbach $\alpha$ values, factor means and standard deviations for the students' approaches to learning mathematics (ALM) questionnaire.

\begin{tabular}{|c|c|c|c|c|}
\hline \multirow{2}{*}{$\begin{array}{c}\text { Item } \\
\text { No. }\end{array}$} & \multicolumn{4}{|c|}{ Factor Loading } \\
\hline & Surface Motive & Surface Strategy & Deep Motive & Deep Strategy \\
\hline 1 & 0.809 & & & \\
\hline 2 & 0.768 & & & \\
\hline 3 & 0.669 & & & \\
\hline 4 & & 0.736 & & \\
\hline 5 & & 0.674 & & \\
\hline 6 & & 0.652 & & \\
\hline 7 & & 0.643 & & \\
\hline 8 & & 0.519 & & \\
\hline 9 & & & 0.795 & \\
\hline 10 & & & 0.765 & \\
\hline 11 & & & 0.756 & \\
\hline 12 & & & 0.750 & \\
\hline 13 & & & 0.513 & \\
\hline 14 & & & 0.502 & \\
\hline 15 & & & & 0.705 \\
\hline 16 & & & & 0.659 \\
\hline 17 & & & & 0.646 \\
\hline$\alpha$ & 0.68 & 0.68 & 0.81 & 0.67 \\
\hline Item Mean \pm SD & $3.54 \pm 0.91$ & $2.96 \pm 0.82$ & $3.34 \pm 0.84$ & $3.57 \pm 0.81$ \\
\hline
\end{tabular}

For students' approaches to learning mathematics, as shown in Table 4, students attained high mean scores for "deep strategy" and "surface motive". Their mean scores for "surface strategy" were lower than those of the other three factors for ALM. Such results suggest that junior secondary school students in Mainland China tend to hold relatively deep approaches to learning mathematics but with mixed motives for learning mathematics.

\subsection{Correlation Analysis}

To explore the relationship between students' COLM and their ALM, a Pearson correlation analysis based on their responses to COLM and ALM was performed. Table 5 summarizes the results. As can be seen in Table 5, statistically significant positive correlations were identified between two factors 
of lower-level conceptions, "memorizing" and "testing", and the two factors of surface approaches: surface motive and surface strategy. However, rather weak or even negative correlations were identified between the two factors "memorizing" and "testing" and the two factors of deep approaches. The third factor of lower-level conceptions, "calculating and practicing", was found to be significantly and positively correlated with the factors in surface approaches and deep approaches.

Table 5. Correlations between the scales of COLM and ALM $(n=1590)$.

\begin{tabular}{ccccc}
\hline Scale & Surface Motive & Surface Strategy & Deep Motive & Deep Strategy \\
\hline Memorizing & $0.29^{* * *}$ & $0.49^{* * *}$ & $0.14^{* * *}$ & $0.05^{*}$ \\
Testing & $0.26^{* * *}$ & $0.53^{* * *}$ & $-0.10^{* * *}$ & $-0.08^{* *}$ \\
Calculating and practicing & $0.31^{* * *}$ & $0.23^{* * *}$ & $0.26^{* * *}$ & $0.27^{* * *}$ \\
Increasing knowledge & $0.29^{* * *}$ & $0.13^{* * *}$ & $0.39^{* * *}$ & $0.34^{* * *}$ \\
Applying & $0.28^{* * *}$ & $0.11^{* * *}$ & $0.36^{* * *}$ & $0.37^{* * *}$ \\
Understanding and & $0.36^{* * *}$ & $0.09^{* * *}$ & $0.56^{* * *}$ & $0.53^{* * *}$ \\
mathematical thinking & & & \\
\hline
\end{tabular}

Note: ${ }^{*} p<0.05,{ }^{* *} p<0.01,{ }^{* * *} p<0.001$.

Moreover, significantly positive correlations were identified between the three factors of higher-level conceptions of learning mathematics, "increasing knowledge", "applying", and "understanding and mathematical thinking", and both the surface approaches and deep approaches. However, as shown in Table 5, the correlation between the higher-level conceptions and deep approaches is stronger than the correlation between the higher-level conceptions and surface approaches.

\subsection{The Stepwise Regression}

A series of stepwise regression analyses were conducted in the study, which used students' conceptions of learning mathematics to predict their approaches to learning mathematics. Table 6 summarizes the regression analysis results. As shown in Table 6, for students' surface approaches to learning mathematics, "testing" could significantly and positively predict "surface motive" and "surface strategy". "Calculating and practicing" made significant predictions for "surface motive", and "memorizing" made positive predictions for "surface strategy". Interestingly, "understanding and mathematical thinking" made quite strong and positive predictions for "surface motive".

Regarding students' deep approaches to learning mathematics, "understanding and mathematical thinking" could positively predict both "deep motive" and "deep strategy", and "applying" could also make positive predictions for "deep motive" and "deep strategy". In the meantime, "calculating and practicing" could positively and significantly predict both "deep motive" and "deep strategy". "Memorizing" also made positive predictions for "deep motive". Lastly, "testing" was the only factor found to make negative predictions for "deep motive".

In summary, these findings indicate that students' lower-level conceptions of learning mathematics, such as "testing" and "memorizing", were more likely to make a positive prediction for students' surface approaches to learning. Relatively speaking, students' higher-level conceptions of learning mathematics made strong predictions for students' deep approaches to learning mathematics. However, surprisingly, several factors of students' lower-level conceptions of learning mathematics, such as "calculating and practicing" and "memorizing", could also positively predict students' deep approaches to learning mathematics. 
Table 6. Stepwise regression model of predicting students' approaches to learning mathematics.

\begin{tabular}{|c|c|c|c|c|c|c|}
\hline $\begin{array}{c}\text { Approaches to Learning } \\
\text { Mathematics }\end{array}$ & Predictors & B & S.E. & $\beta$ & $\mathbf{T}$ & $\mathbf{R}^{2}$ \\
\hline \multicolumn{7}{|l|}{ Surface motive } \\
\hline & Understanding and mathematical thinking & 0.41 & 0.03 & 0.33 & $13.80 * * *$ & 0.23 \\
\hline & Testing & 0.27 & 0.02 & 0.26 & $11.26^{* * *}$ & \\
\hline & Practicing and exercise & 0.16 & 0.03 & 0.14 & $5.72 * *$ & \\
\hline & Constant & 0.69 & 0.13 & & $5.28 * *$ & \\
\hline \multirow[t]{4}{*}{ Surface strategy } & & & & & & 0.34 \\
\hline & Testing & 0.33 & 0.02 & 0.36 & $14.77^{* * *}$ & \\
\hline & Memorizing & 0.27 & 0.02 & 0.29 & $11.80 * * *$ & \\
\hline & Constant & 1.24 & 0.06 & & $19.59^{* * *}$ & \\
\hline \multicolumn{7}{|l|}{ Deep motive } \\
\hline & Understanding and mathematical thinking & 0.53 & 0.03 & 0.47 & $19.04 * * *$ & 0.34 \\
\hline & Applying & 0.10 & 0.02 & 0.10 & $3.99 * *$ & \\
\hline & Testing & -0.13 & 0.02 & -0.14 & $-5.45^{* * *}$ & \\
\hline & Memorizing & 0.10 & 0.03 & 0.10 & $3.76^{* *}$ & \\
\hline & Calculating and practicing & 0.07 & 0.03 & 0.06 & $2.61 * *$ & \\
\hline & Constant & 0.96 & 0.11 & & $8.50^{* * *}$ & \\
\hline \multicolumn{7}{|l|}{ Deep strategy } \\
\hline & Understanding and mathematical thinking & 0.51 & 0.03 & 0.45 & $18.27^{* * *}$ & 0.30 \\
\hline & Applying & 0.13 & 0.02 & 0.13 & $5.11 * * *$ & \\
\hline & Calculating and practicing & 0.10 & 0.02 & 0.09 & $3.81 * *$ & \\
\hline & Constant & 1.14 & 0.11 & & $10.47^{* * *}$ & \\
\hline
\end{tabular}

Note: ${ }^{*} p<0.05,{ }^{* *} p<0.01,{ }^{* * *} p<0.001$.

\section{Discussion}

The main goal of the study is to investigate the characteristics of Chinese students' conceptions of and approaches to learning mathematics. Firstly, two widely-used questionnaires (COLM and ALM) were modified in the field of mathematics education and the validity and reliability (e.g., factor loading and Cronbach's alpha values) of the two questionnaires were examined by EFA and CFA. Both the EFA and CFA results as reported above displayed a similar factor structure, as identified in previous studies $[10,16]$, with satisfactory reliability. In particular, with respect to COLM, the six extracted factors in the study imply a similar factor structure of students' conceptions of learning mathematics, as revealed in previous studies in science-related subjects [16,22]. As shown in Table 3, while the students' scored higher on the three factors of higher-level conceptions, they scored lower on the first two factors of lower-level conceptions, "memorizing" and "testing". These results reveal that the surveyed Chinese junior secondary school students tend to view learning mathematics from a constructivist and higher-level perspective $[14,16]$.

However, unlike results found in previous studies [22], the mean score of the other factor of lower-level conceptions, "calculating and practicing", is the highest among all the six factors of COLM. This difference may be due to the different learning domains. Findings in previous studies [10] are from science-related subjects, such as biology or physics. The domain-specific attribute of mathematics may encourage students to conceptualize "calculating and practicing" more [29]. Indeed, calculating and practicing exercises are a basic and important part of learning mathematics well. In addition, due to the pressure of examinations in China, students are often engaged in huge amounts of practice of different skills in solving different types of problems so that they will be able to solve problems quickly [32].

With respect to ALM, as shown at the bottom of Table 4, while the students in the study scored higher for "deep strategy" than "surface strategy", they scored lower for "deep motive" than "surface motive". Such findings illustrate that even though the students tend to use more meaningful strategies to learn mathematics, they still hold quite strong surface motives to learn mathematics. This result is similar to findings identified in previous studies [22]. 
The second main purpose of the study is to investigate the relationships between students' conceptions of learning mathematics and their approaches to learning mathematics. In general, the results of the study show that two factors of students' lower-level conceptions, "memorizing" and "testing", are positively correlated with and more powerful in predicting students' surface approaches to learning mathematics and are weakly or negatively correlated with and less powerful in predicting students' deep approaches to learning mathematics. This finding is not consistent with findings identified in previous studies. Previous studies in science-related subjects such as biology and physics found that "testing" only predicted "surface strategy" [16,22]. The finding in the present study suggests that strong conceptions of "memorizing" and "testing" tend to cause students to employ more rote-learning approaches in learning mathematics, which are considered to be important for examination.

In addition, the Person correlation analysis and stepwise regression analysis results show that students who hold stronger higher-level conceptions of learning mathematics tend to have a deep motive and to use deep strategies in learning mathematics. This finding is consistent with the associations between students' conceptions of and approaches to learning identified in other school subjects (such as in physics; e.g., Chiou et al. [22]). This finding also supports the argument made by previous researchers that a constructive conception of learning will foster deep approaches to learning $[5,16]$.

However, there are several relationships which contradict the "lower-level versus surface approach" and "higher-level versus deep approach" relationships as theoretically argued by previous researchers. First, "calculating and practicing" has quite a strong predictive power for both surface and deep approaches to learning, which is quite different from findings identified in previous studies [10,22]. For example, Chiou et al. found that "calculating and practicing" in Taiwanese students' conception of learning physics only positively predicted students' surface motive [22]. This inconsistency may be explained by the differences in the two school subjects. Although learning physics also involves quite a large number of exercises, it involves quite a large number of experiments as well. However, for the learning of mathematics at the secondary school level, calculating and practicing is the most predominant method to improve their understanding and enhance their problem-solving skills and speed [32].

Secondly, students' higher-level conception, "understanding and mathematical thinking", has quite a strong predictive power for students" "surface motive" in addition to "deep motive" and "deep strategy", which is similar to findings in previous studies [30]. Findings like this illustrate that although the conception of "understanding and mathematical thinking" tends to make students use more constructive-oriented learning strategies, the motivation beneath this strategy may not be persistent. Again, this may be due to the influence of examination pressure in Mainland China. In Mainland China, students need to take examinations frequently even at the junior secondary school level. This suggests that even though students sometimes hold higher-level conception of learning mathematics, they may not have deep motive in learning mathematics due to the influences or pressure that come with the context in which the mathematics learning takes place.

\section{Conclusions and Implication}

Mathematics plays an important role in students' long-term development [11]. Therefore, a clear understanding of students' conceptions of and approaches to learning mathematics will contribute to the interpretation of students' outcomes in mathematics. The present study is one of the few studies in mathematics education that investigates junior school students' conceptions of and approaches to learning mathematics and their relationship in Mainland China. Therefore, findings of the study not only contribute to the understanding of the Chinese Learner Paradox, but also the understanding of the relationship between students' conception of and approaches to learning mathematics in the field of mathematics education and in the social and cultural context in China. 
Using a quantitative method, the findings as reported above suggest that Chinese junior school students tend to hold higher-level conceptions of learning mathematics rather than lower-level conceptions such as memorizing and testing. The students tend to hold a rather mixed surface and deep motive in learning mathematics, but they are more likely to employ deep approaches to learning mathematics. The findings of the present study also confirm the structural relationship between students' conceptions of and approaches to learning in the subject of mathematics and in Mainland China. Two factors of students' lower-level conceptions of learning mathematics, "memorizing" and "testing", were the strongest predictor for the surface approaches to learning mathematics, while students' higher-level conceptions of learning mathematics, such as "applying" and "understanding and mathematical thinking", had a noticeable effect on their deep approaches to learning mathematics. In the meantime, the influence of the social and cultural context is also found to be a critical factor which affects the relationship between students' conceptions of and approaches to learning mathematics. Thus, the findings of this study support the assertion that students' conceptions of and approaches to learning are socially and culturally dependent $[9,10]$.

In terms of practical implications, firstly, the findings of the study suggest that, in practice, mathematics educators could make attempts to foster students' higher-level conceptions of learning mathematics, such as "applying" and "understanding and mathematical thinking", with the aim of facilitating students' deep mathematical learning approaches. Secondly, the two questionnaires developed in this study have high reliability and validity. Mathematics education researchers, especially mathematics education researchers in Mainland China, could therefore consider using these two questionnaires to explore students' conceptions of and approaches to learning mathematics at other grade levels.

The study also has several limitations. Firstly, the study only used questionnaire surveys to investigate students' conceptions of and approaches to learning mathematics. Future studies in this area could use other research methods such as interviews to reach a deeper and fuller understanding of Chinese students' conceptions of and approaches to leaning mathematics. Secondly, the study only investigated students at the junior secondary school level in Mainland China. To achieve a fuller understanding of this relationship in mathematics education, more studies conducted with students at other schooling stages and in other cultural and social contexts are needed. Thirdly, the study did not explore the development or change process of students' conceptions of and approaches to learning mathematics. Future studies could consider employing a longitudinal study design to investigate the development process of students' conceptions of and approaches to learning mathematics and the relationships between these two constructs.

Author Contributions: Conceptualization, X.Y. and F.L.; Methodology, X.Y. and S.Z.; Formal Analysis, X.Y. and S.Z.; Investigation, X.Y.; Resources, X.Y.; Writing-Original Draft Preparation, X.Y.; Writing-Review \& Editing, X.Y. and F.L.; Project Administration, S.Z.; Funding Acquisition, X.Y.

Funding: This study was funded by The Fundamental Research Funds for the Central Universities (XDJK2016B013).

Conflicts of Interest: The authors declare no conflict of interest.

\section{References}

1. Organization for Economic Co-operation and Development. PISA 2015 Results (Volume I): Excellence and Equity in Education; OECD Publications: Paris, France, 2016.

2. Stevenson, H.W.; Lee, S.Y.; Chen, C.; Lummis, M.; Stigler, J.; Fan, L.; Ge, F. Mathematics achievement of children in China and the United States. Child Devel. 1990, 61, 1053-1066. [CrossRef]

3. Leung, F.K. In search of an East Asian identity in mathematics education. Educ. Stud. Math. 2001, 47, 35-51. [CrossRef]

4. Ni, Y.; Zhou, D.; Li, X.; Li, Q. Relations of Instructional Tasks to Teacher-Student Discourse in Mathematics Classrooms of Chinese Primary Schools. Cognit. Instr. 2014, 32, 2-43. [CrossRef]

5. Biggs, J. Learning from the Confucian heritage: so size doesn't matter? Int. J. Educ. Res. 1998, 29, 723-738. [CrossRef] 
6. Ni, Y.J.; Chiu, C.C.; Chan, Z.J. Chinese children learning mathematics: From home to school. In The Handbook of Chinese Psychology; Bond, M.H., Ed.; Oxford University Press: Oxford, UK, 2010; pp. 143-154.

7. Wei, D. Parental influence on Chinese students' achievement: A social capital perspective. Asia Pac. J. Educ. 2012, 32, 153-166. [CrossRef]

8. Ellis, R.A.; Goodyear, P.; Calvo, R.A.; Prosser, M. Engineering students' conceptions of and approaches to learning through discussions in face-to-face and online contexts. Learning Instr. 2008, 18, 267-282. [CrossRef]

9. Biggs, J.; Kember, D.; Leung, D.Y. The revised two-factor study process questionnaire: R-SPQ-2F. British J. Educ. Psychol. 2001, 71, 133-149. [CrossRef]

10. Chiou, G.L.; Liang, J.C.; Tsai, C.C. Undergraduate students' conceptions of and approaches to learning in biology: A study of their structural models and gender differences. Int. J. Sci. Educ. 2012, 34, 167-195. [CrossRef]

11. Papadakis, S.; Kalogiannakis, M.; Zaranis, N. Improving Mathematics Teaching in Kindergarten with Realistic Mathematical Education. Early Child. Educ. J. 2016, 45, 369-378. [CrossRef]

12. Sterling, S.; Thomas, I. Education for sustainability: The role of capabilities in guiding university curricula. Int. J. Innov. Sustain. Devel. 2006, 1, 349-369. [CrossRef]

13. Kember, D.; Watkins, D. Approaches to learning and teaching by the Chinese. In The Second Oxford Handbook of Chinese Psychology; Bond, M.H., Ed.; Oxford University Press: Oxford, UK, 2010; pp. 169-185.

14. Tsai, C.C. Conceptions of learning science among high school students in Taiwan: A phenomenographic analysis. Int. J. Sci. Edu. 2004, 26, 1733-1750. [CrossRef]

15. Rao, N.; Chan, C.K.K. Moving beyond paradoxes: Understanding Chinese learners and their teachers. In Revisiting the Chinese Learner: Changing Contexts, Changing Education; Comparative Education Research Centre/Springer Academic Publishers: Hong Kong, China, 2010; pp. 3-31.

16. Lee, M.H.; Johanson, R.E.; Tsai, C.C. Exploring Taiwanese high school students' conceptions of and approaches to learning science through a structural equation modeling analysis. Sci. Educ. 2008, 92, 191-220. [CrossRef]

17. Liang, J.C.; Tsai, C.C. Relational analysis of college science-major students' epistemological beliefs toward science and conceptions of learning science. Int. J. Sci. Educ. 2010, 32, 2273-2289. [CrossRef]

18. Saljo, R. Learning in the Learner's Perspective. I. Some Common-Sense Conceptions; Institute of Education, University of Gothenburg: Gothenburg, Sweden, 1979. Available online: https://eric.ed.gov/?id=ED173369 (accessed on 27 April 2019).

19. Chiu, M.S. Identification and assessment of Taiwanese children's conceptions of learning mathematics. Int. J. Sci. Math. Educ. 2012, 10, 163-191. [CrossRef]

20. Reid, A.; Wood, L.N.; Smith, G.H.; Petocz, P. Intention, approach and outcome: University mathematics students' conceptions of learning mathematics. Int. J. Sci. Math. Educ. 2005, 3, 567-586. [CrossRef]

21. Lin, C.L.; Tsai, C.C.; Liang, J.C. An investigation of two profiles within conceptions of learning science: An examination of confirmatory factor analysis. Eur. J. Psychol. Educ. 2012, 27, 499-521. [CrossRef]

22. Chiou, G.L.; Lee, M.H.; Tsai, C.C. High school students' approaches to learning physics with relationship to epistemic views on physics and conceptions of learning physics. Res. Sci. Technol. Educ. 2013, 31, 1-15. [CrossRef]

23. Peterson, E.R.; Brown, G.T.; Irving, S.E. Secondary school students' conceptions of learning and their relationship to achievement. Learning Individ. Differ. 2010, 20, 167-176. [CrossRef]

24. Kember, D.; Biggs, J.; Leung, D.Y. Examining the multidimensionality of approaches to learning through the development of a revised version of the Learning Process Questionnaire. British J. Educ. Psychol. 2004, 74, 261-279. [CrossRef]

25. Biggs, J.B. Approaches to learning in secondary and tertiary students in Hong Kong: Some comparative studies. Educ. Res. J. 1991, 6, 27-39.

26. Duarte, A.M. Conceptions of learning and approaches to learning in Portuguese students. Higher Educ. 2007, 54, 781-794. [CrossRef]

27. Zhu, C.; Valcke, M.; Schellens, T. A cross-cultural study of Chinese and Flemish university students: Do they differ in learning conceptions and approaches to learning? Learning Individ. Differ. 2008, 18, $120-127$. [CrossRef]

28. Li, W.T.; Liang, J.C.; Tsai, C.C. Relational analysis of college chemistry-major students' conceptions of and approaches to learning chemistry. Chem. Educ. Res. Pract. 2013, 14, 555-565. [CrossRef] 
29. Shen, K.M.; Lee, M.H.; Tsai, C.C.; Chang, C.Y. Undergraduate students' earth science learning: Relationships among conceptions, approaches, and learning self-efficacy in Taiwan. Int. J. Sci. Educ. 2016, 38, 1527-1547. [CrossRef]

30. Liang, J.C.; Su, Y.C.; Tsai, C.C. The assessment of Taiwanese college students' conceptions of and approaches to learning computer science and their relationships. Asia-Pacific Educ. Res. 2015, 24, 557-567. [CrossRef]

31. Wang, Y.L.; Liang, J.C.; Lin, C.Y.; Tsai, C.C. Identifying Taiwanese junior-high school students' mathematics learning profiles and their roles in mathematics learning self-efficacy and academic performance. Learning Individ. Differ. 2017, 54, 92-101. [CrossRef]

32. Cai, J.; Nie, B. Problem solving in Chinese mathematics education: Research and practice. ZDM-The Int. J. Math. Educ. 2007, 30, 459-473. [CrossRef]

(C) 2019 by the authors. Licensee MDPI, Basel, Switzerland. This article is an open access article distributed under the terms and conditions of the Creative Commons Attribution (CC BY) license (http://creativecommons.org/licenses/by/4.0/). 